\title{
Potencialidades de las TIC y su papel fomentando la creatividad: percepciones del profesorado
}

\section{(ICT potentials and their role in promoting creativity: teachers' perceptions)}

\author{
María José Cuetos Revuelta \\ Universidad Internacional de la Rioja, UNIR (España) \\ Lucía Grijalbo Fernández \\ Elena Argüeso Vaca \\ Vanessa Escamilla Gómez \\ Ruth Ballesteros Gómez \\ Bureau Veritas Formación (España)
}

DOI: http://dx.doi.org/10.5944/ried.23.2.26247

\section{Cómo referenciar este artículo:}

Cuetos Revuelta, M. J., Grijalbo Fernández, L., Argüeso Vaca, E., Escamilla Gómez, V., y Ballesteros Gómez, R. (2020). Potencialidades de las TIC y su papel fomentando la creatividad: percepciones del profesorado. RIED. Revista Iberoamericana de Educación a Distancia, 23(2), pp. 287-306. doi: http:// dx.doi.org/10.5944/ried.23.2.26247

\section{Resumen}

Las Tecnologías de la Información y la Comunicación (TIC) ofrecen nuevas oportunidades de aprendizaje en una sociedad interconectada, donde su uso se ha hecho fundamental. Esta investigación presenta los resultados de las percepciones de 245 profesores que están cursando un máster online de eLearning y Tecnología Educativa sobre las potencialidades de las TIC más apreciadas dentro del ámbito educativo, así como el papel de las nuevas tecnologías en el fomento de la creatividad de los estudiantes. Los participantes también valoraron sus competencias de manejo de las TIC y su propia creatividad. Este enfoque permite profundizar en las consideraciones de los participantes desde su propia percepción y contexto.

Los resultados apuntan a que los docentes atribuyen a las TIC una alta motivación y capacidad de estimulación. Las catalogan como un importante canal de comunicación e intercambio de información, así como medio de expresión y creación. Destacan que la orientación de profesores y padres en el manejo de las TIC es básica para trabajar con recursos digitales, debiéndose fomentar la competencia digital docente (CDD). 
Se concluye que para potenciar la creatividad de los estudiantes no basta con utilizar únicamente las TIC, hay que trabajar el pensamiento divergente mediante la combinación de métodos y actividades. Se debe aprovechar lo mejor de cada metodología para hacer la formación lo más motivadora posible y, así, construir un ambiente de aprendizaje que estimule la creatividad de manera más efectiva a través de las interacciones entre los alumnos y estas herramientas de aprendizaje.

Palabras clave: creatividad; motivación; aprendizaje cooperativo; tecnología de la información.

\begin{abstract}
Information and Communication Technologies (ICT) offer new learning opportunities in an interconnected society, where its use has become fundamental. This study presents the results of the perceptions of 245 professors who are studying an online master's degree in eLearning and Educational Technology about the potential of the most valued ICT within the educational field and the role of new technologies in the promotion of creativity of the students is shown. Participants also self-assess their ICT management competence and their own creativity. This approach allows to deepen in the considerations of the participants from their own perception and context.

The results suggest that teachers attribute high motivation and stimulation capacity to ICT, categorizing them as an important communication and information exchange channel as well as a way of expression and creation. They emphasized that the orientation of teachers and parents in handling ICT is essential to work with digital resources and the digital teaching competence (DTC) should be encouraged.

It is concluded that to enhance students' creativity, the mere use of ICT does not suffice, but divergent thinking must be worked through the combination of methods and activities. The best of each methodology should be used to make the training as motivating as possible and, thus, create a learning environment that stimulates creativity more effectively through interactions between students and these learning tools.
\end{abstract}

Keywords: creativity; motivation; cooperative learning; information technology.

Las generaciones nacidas en el nuevo siglo han convivido desde siempre con las Tecnologías de la Información y la Comunicación (TIC) y conciben su presencia como algo natural en su desarrollo, para obtener información o mantenerse en contacto con familiares y amigos. Las TIC han producido cambios en los hábitos de conducta y en el modo de relacionarnos (Maldonado, García y Sampedro-Requena, 2019).

El uso de las TIC en educación puede mejorar la calidad del proceso de enseñanzaaprendizaje. A priori, parece que un entorno de aprendizaje basado en las TIC ayuda a que ideas difíciles se hagan más comprensibles, por lo que los estudiantes construyen sus conocimientos de manera que sean significativos para ellos. Una de sus principales ventajas es la motivación de los alumnos, ya que el aprendizaje 
les resulta más atractivo y divertido, lo que hace que los estudiantes dediquen más tiempo al estudio y se encuentren más implicados en todas las actividades (Ferro Soto, Martínez Senra y Otero Neira, 2009). Otras ventajas son la ruptura de las barreras espacio-temporales y la gran cantidad de recursos disponibles (Talebian, Mohammadi y Rezvanfar, 2014), de manera que la adquisición de conocimientos no se ve restringida al aula. Estas tecnologías permiten adaptar los contenidos a las necesidades y características del alumnado, proporcionándoles oportunidades para explorar y aprender a su propio ritmo y tiempo (Phutela y Dwivedi, 2019). Se desarrolla así una enseñanza centrada en el alumno (Das, 2019), a pesar de que el docente necesite mucho más tiempo para preparar los contenidos TIC que va a trabajar con los estudiantes. Las tecnologías e Internet han posibilitado prácticas abiertas, lo que abre un espectro de posibilidades de producción de recursos y uso en ámbitos sociales y culturales para la creación colectiva y, con ello, la posibilidad de trabajar con saberes compartidos (Ramírez-Montoya y García-Peñalvo, 2018). La interacción social y la comunicación son el terreno común del aprendizaje colaborativo y el aumento de la creatividad (Lappas y Fessakis, 2014).

El marco estratégico de Educación y Formación 2020 (ET2020) busca el desarrollo de una educación y una formación abiertas e innovadoras para lograr una plena incorporación en la era digital, manteniendo como uno de sus objetivos estratégicos incrementar la creatividad y la innovación. La creatividad se posiciona como una parte vital de la educación para el futuro digital y para los entornos de aprendizaje impulsados por la tecnología y la vida laboral con la que los estudiantes inevitablemente se enfrentarán (Creely, Henderson y Henriksen, 2019). Sin embargo, aspectos como la multiculturalidad, la digitalización de la información y la importancia de las redes sociales no se abordan con suficiente importancia en nuestras aulas, siendo aún un recurso infrautilizado en la enseñanza (Tabuenca, Sánchez y Cuetos, 2019).

Aunque la creatividad se ha convertido en un tema central para la enseñanza y el aprendizaje del siglo XXI, todavía no está claro qué significa esto para el campo de la educación, en política y, por lo tanto, en la práctica. Se hace necesario un enfoque más coherente entre la implementación de la creatividad y la tecnología en la teoría de las políticas educativas y la realidad de las aulas (Henriksen, Henderson, Creely, Ceretkova, Černochová, Sendova, Sointu y Tienken, 2018). Esto puede ser debido a que es difícil aproximarse a una definición concreta de creatividad. Hay diferentes percepciones de su significado en función de las técnicas existentes para su evaluación. En los orígenes se describió como la habilidad que se posee para resolver preguntas y problemas (Smilansky y Halberstadt, 1986); una habilidad intelectual para generar un producto original o con un valor añadido, social o personal, con un propósito concreto y usando una información dada (Hu y Adey, 2002). La creatividad se asocia a la generación de nuevas ideas lo que involucra un complejo sistema funcional que permiten al individuo recibir, analizar, comparar y generar ideas o respuestas (Sarmiento, 2017). Hay cuatro rasgos concurrentes que 
varios autores coinciden como distintivos de la creatividad: fluidez, flexibilidad, originalidad y elaboración (Lappas y Fessakis, 2014; Moguel Pérez, Michel López y Torres Hernández, 2016). Se refieren a la producción de múltiples ideas o alternativas, su procesamiento de formas variadas, rompiendo con la rutina, pero de un modo estructurado tras un proceso de síntesis. Más recientemente, Nikolopoulou (2018) realizó una recopilación de la interacción entre las características de las TIC y las de la creatividad, añadiendo conceptos como: uso de la imaginación, originalidad y pensamiento crítico y divergente.

Así, la creatividad puede promoverse y extenderse con el uso de nuevas tecnologías donde se brinden oportunidades para el desarrollo de ideas, hacer conexiones, crear y hacer y fomentar estrategias como la colaboración, comunicación y evaluación (Loveless, 2002). Parece que el uso de las TIC en programas de mejora de la creatividad da resultados positivos (Rashid y Rahman, 2014; Stolaki y Economides, 2018; Sokól, Figurska y Blasková, 2015) ya que la integración de las TIC en el método de enseñanza y el diseño de las actividades es propicia para la expresión de la creatividad de los estudiantes y su habilidad para la resolución de problemas. Muchos estudios apoyan la noción de que el aprendizaje electrónico, el intercambio de conocimientos en línea y la interactividad mejoran la creatividad de los estudiantes (Phutela y Dwivedi, 2019; Wei, Peng y Chou, 2015; Yeh, Yeh, y Chen, 2012). Sin embargo, la creatividad está profundamente relacionada con los problemas de integración de la tecnología, por lo que estos problemas de creatividad y tecnología pueden considerarse en conjunto (Henriksen, Mishra y Fisser, 2016).

Es importante tener en cuenta que no es en las propias TIC en las que hay que buscar la clave para entender su impacto sobre la educación, sino en las posibilidades que éstas ofrecen para la interacción, la participación y la demostración activa de imaginación, producción, propósito, originalidad y valor, tanto a los profesores como a los estudiantes (Coll, 2011; Nikolopoulou, 2018). Para que el uso de las TIC sea el más adecuado, docentes y familias juegan una función esencial como factores extrínsecos, destacando la influencia de los profesores en la creatividad de sus estudiantes, aunque sean ya adultos (Ehtiyar y Baser, 2019). Estas acciones necesitan un profesorado formado en este ámbito, que involucre a las TIC en la enseñanza de su alumnado y les oriente en su uso para maximizar las posibilidades de producción creativa (Giménez, Luengo y Bartrina, 2017; Stolaki y Economides, 2018, Henriksen, Mishra y Fisser, 2016), mejorando la actividad docente y el aprendizaje de sus estudiantes. Actualmente, la zanja más profunda tiene otro carácter, el generacional, al menos en el marco europeo, pues los estudiantes tienen más habilidades en el manejo de las herramientas de aprendizaje y acceso a fuentes de conocimiento que sus educadores, padres o tutores.

Los docentes deben ser, por tanto, los responsables de crear en el aula un ambiente que permita a los estudiantes participar en un entorno diverso, utilizando una variada gama de estrategias de enseñanza, búsqueda, organización y selección de información, indagación y fomento del pensamiento creativo, entre otras 
(Betancourt Morejón y Valadez Sierra, 2009). Es indispensable encontrar puntos de unión entre creatividad y educación y, para ello, hay que crear espacios alternativos, diversos y estimulantes (Jorda Lueges y Martínez Vázquez, 2015).

Resulta fundamental entender el papel de los docentes en el apoyo al desarrollo de la creatividad en las aulas. Señalar aquí, la contribución de este estudio para conocer la percepción en una pequeña muestra de profesores, con base en su visión y experiencia docente, sobre qué potencialidades de las TIC son las que más valoran dentro del ámbito educativo y qué papel tienen las nuevas tecnologías en el fomento de la creatividad de los discentes.

\section{METODOLOGÍA DE LA INVESTIGACIÓN}

\section{Contexto del estudio}

La muestra de estudio estaba formada por docentes en activo en centros de todos los niveles educativos que eran a su vez estudiantes del Máster Oficial en eLearning y Tecnología Educativa, máster de modalidad online (eLearning) de Bureau Veritas Formación.

En concreto, se analizaron las respuestas de un total de 245 alumnos de este máster en 4 convocatorias consecutivas, correspondientes a los cursos 2015/16, 2016/17, 2017/18 y 2018/19. El número de estudiantes en cada caso fue de 56, 46, 132 y 21 , respectivamente.

\section{Participantes}

Del total de los 245 participantes, el 66\% eran mujeres (161 alumnas) y el 34\% restante eran hombres (84 alumnos). De ellos, un $97 \%$ eran de diversos puntos del ámbito nacional, existiendo sólo 8 alumnos extranjeros (procedentes de Colombia, Chile y Ecuador), por ello esta característica no se ha considerado relevante para el análisis de los resultados obtenidos.

Las edades de los participantes estaban comprendidas entre los 23 y los 52 años. La edad media fue de 32.6 años con una desviación estándar de 6.1, existiendo sólo 4 profesores con edad superior o igual a los 50 años.

Para tener más información sobre las características de los participantes (y en concreto tener información sobre su propia creatividad) se recurre al análisis de un cuestionario de autoevaluación de las competencias profesionales docentes, donde ellos mismos valoraron una serie de aspectos sobre sus habilidades y capacidades, en rango de o a 5 . Marcando o si no poseían conocimientos y 5 si tenía control y dominio sobre el tema evaluado. Los ítems estaban agrupados en bloques, el primero relacionado con su propia competencia digital (habilidades básicas del manejo de las TIC), el segundo sobre iniciativa y confianza en sí mismo (iniciativa y espíritu 
emprendedor) y el resto relacionados de algún modo con las diversas definiciones de la creatividad vistas en el marco teórico (capacidad para resolver problemas de forma colaborativa, capacidad para diseñar soluciones alternativas a problemas y capacidad de generar nuevas ideas).

Para este artículo los datos se trataron de forma anónima y se analizaron por grupos de edad para saber si existía similitud en las medias obtenidas para cada uno de los aspectos analizados.

\section{Recogida y análisis de datos}

Se optó por una metodología de carácter cualitativo con el fin de explorar las concepciones de los participantes de una forma abierta con base en sus experiencias y testimonios en los centros escolares. La técnica de recogida de información fue a través de un foro online, dentro del marco de la asignatura "Tecnologías y Entornos Virtuales para eLearning" que cursaban en el máster. Podían participar exponiendo sus puntos de vista una o varias veces a lo largo de la duración de la asignatura justificando sus respuestas e interactuando con sus compañeros. El foro se mantuvo abierto durante los 2 meses de duración de la asignatura en cada curso y el 100\% del alumnado participó en al menos una ocasión. Las dos principales preguntas de la investigación fueron:

- “ilas herramientas tecnológicas ayudan a potenciar la creatividad de los estudiantes?”

- “¿qué potencialidades de las TIC son las que más valoras dentro del ámbito educativo y cómo las relacionas con la creatividad?”

En primer lugar, se llevó a cabo el análisis del contenido de las respuestas de la pregunta central del estudio sobre si las herramientas tecnológicas ayudan a potenciar la creatividad de los estudiantes. Posteriormente, de modo inductivo se extrapolaron de la información de las respuestas de los foros las potencialidades de las TIC, con base en la justificación dada. Se creó un sistema de categorías de las características específicas de las herramientas TIC que se puedan correlacionar posteriormente con las características básicas de la creatividad (elementos de los procesos creativos), siguiendo una adaptación de las aportadas por la revisión bibliográfica del estudio de Nikolopoulou (2018). Las categorías seleccionadas han sido:

- Motivación

- Inmediatez en el acceso a la información

- Flexibilidad

- Fomento del aprendizaje colaborativo

- Medio de expresión y creación 
- Nuevos espacios interactivos y formas de presentar la información

- Fuente de dinamismo

- Canal de comunicación e intercambio

Este proceso inductivo de categorización se ha implementado de modo simultáneo por dos investigadores diferentes, obteniéndose un alto grado de concordancia en las frecuencias de las categorías seleccionadas de los resultados finales. El análisis de los datos se llevó a cabo mediante el programa EXCEL 2018 de Microsoft Office.

\section{RESULTADOS}

\section{Competencias profesionales docentes}

En la figura 1 se puede observar la superposición de las medias de las autoevaluaciones del profesorado partícipe en el estudio. Los datos mostraron cómo las valoraciones fueron relativamente altas en todos los casos (entre 3 y 4), siendo prácticamente coincidentes para todos los ítems en los encuestados de igual o menos de 30 años.

Pero lo relevante del análisis fue comprobar cómo la valoración de todas las características disminuía con el aumento en la edad de los encuestados. El dato más significativo fue el del ítem relacionado con el uso o manejo de las TIC, que pasó de un valor medio de 3.9 en los profesores menores de 30 años a 3.0 en los de edad mayor o igual a 50 años. La diferencia de edad mostró la existencia de una menor habilidad en estos docentes en lo referido a sus competencias digitales. Este dato resulta curioso ya que no hay que olvidar que estaban cursando estudios de modalidad online. 
Figura 1. Comparativa por edades del análisis de competencias profesionales autoevaluadas por los participantes

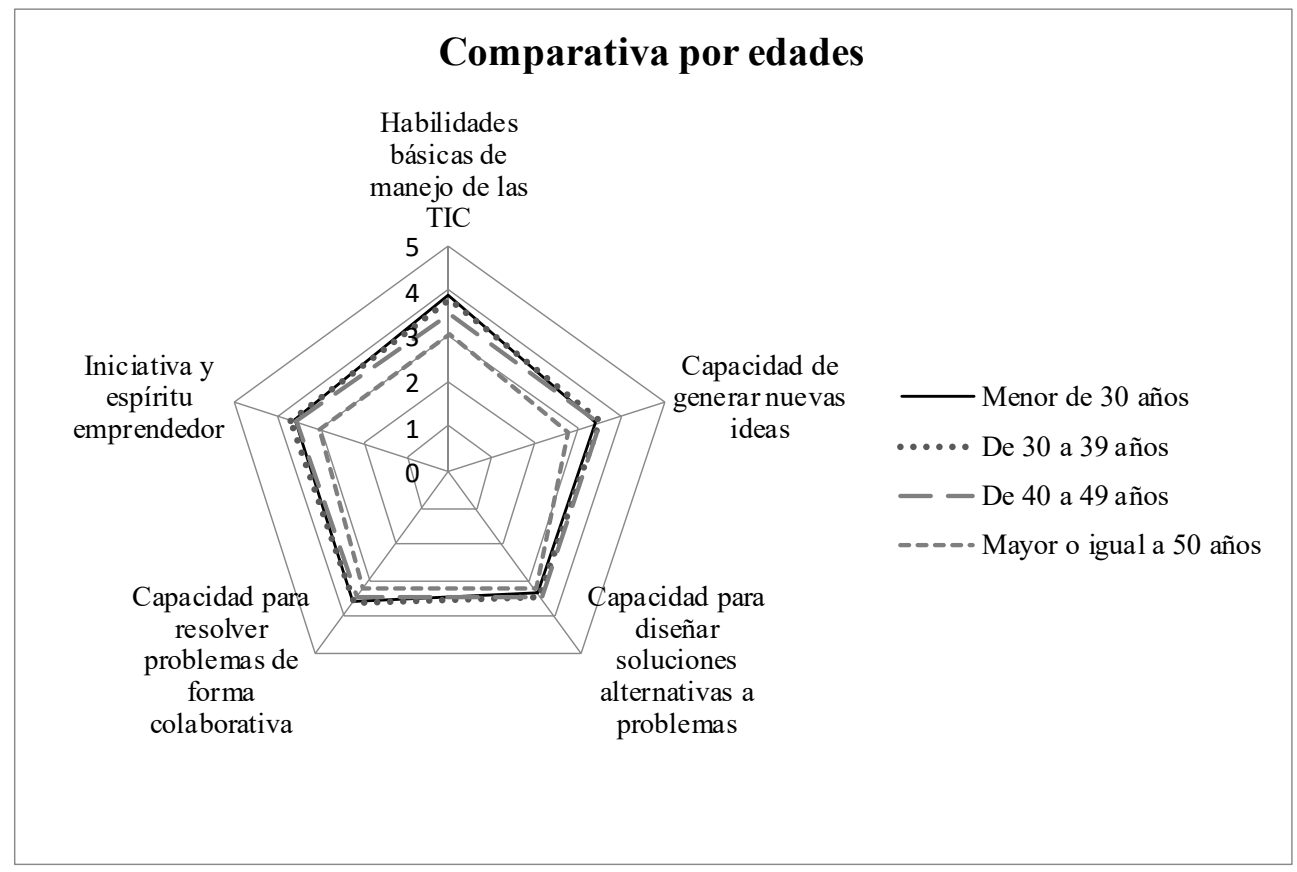

Fuente: Elaboración propia

"La capacidad de generar nuevas ideas" y "la iniciativa y el espíritu emprendedor" fueron los aspectos que más disminuyeron con el aumento en la edad de los participantes, variando entre ambos rangos de edad de 3.4 a 2.7 y de 3.6 a 3 , respectivamente. Finalmente, hay que comentar que el ítem de "la capacidad para diseñar soluciones alternativas a problemas" fue uno de los peor valorados por todos los participantes, independientemente del rango de edad al que pertenecieran. Observándose, por tanto, una disminución en el valor de los temas relacionados con la creatividad a medida que aumentaba la edad del encuestado.

\section{Potencialidades del uso de las TIC}

En una primera aproximación, haciendo un resumen de las respuestas a la primera pregunta del estudio, el 85\% de los profesores que participaron en el foro confirmaron que las TIC fomentan la creatividad de los estudiantes por las múltiples potencialidades que presentan (como se analizará posteriormente), frente a un $2 \%$ que respondieron negativamente. Estos indicaron que incluso pueden llegar a 
generar el efecto de coartar la creatividad, ya que ayudan a simplificar en exceso las tareas, o a disminuir el esfuerzo que se debe aplicar en el proceso de aprendizaje, pudiendo llegar a sobreestimular a los alumnos o a conducirles a un aislamiento social. Como ejemplos se plasman las siguientes referencias textuales:

- "Las TIC no han potenciado la creatividad e incluso en muchos casos nos hacen ser más pasivos. Estos medios están exigiendo mucho menos esfuerzo a la hora de aprender y esto nos puede llevar a la consecuencia de que se generará menos creatividad" (Participante 16, curso 15-16).

- "La tecnología, en ocasiones, nos facilita demasiado la tarea y sistemas como por ejemplo el GPS o los videojuegos hacen que tanto los adultos como los niños tengan que minimizar su esfuerzo y creatividad para realizar una acción tan simple como la de llegar de un punto de una ciudad a otro o el inventar un juego nuevo" (Participante 141, curso 17-18).

- "Vivimos en un mundo cada vez más tecnológico, en el cual hay que tener cuidado para evitar que nuestros jóvenes y nuestros mayores caigan en las garras negativas de la tecnología y esto les lleve a aislarse, a meterse en su mundo virtual y a desvincularse de la realidad" (Participante 113, curso 17-18).

Cabe destacar la existencia de un 13\% de intervenciones que no se decantaron por una opción concreta. Para ello, alegaron que las TIC son sólo herramientas que tenemos a nuestra disposición, que con un mayor o menor grado de importancia podrían ayudar a los estudiantes, pero la creatividad y el talento son capacidades personales. De entre las citas al respecto se pueden destacar:

- "Las TIC no son las únicas herramientas, muchos de nuestros literatos más creativos escribían sus obras a mano y reconocían no manejar ningún tipo de herramienta tecnológica en fase creativa" (Participante 8, curso 15-16).

- "Una persona creativa puede serlo tanto con un lápiz y un papel como con un programa informático. Las herramientas ayudan a potenciar esa creatividad, pero no son factores decisivos" (Participante 30, curso 15-18).

Respecto a la segunda pregunta del estudio, en la figura 2 se recogen los datos del total de referencias textuales analizadas agrupadas en función de las potencialidades otorgadas a las TIC en el ámbito educativo.

La principal potencialidad que los profesores atribuyeron a las TIC fue "la motivación y la estimulación de los estudiantes", citada 88 veces. Se incluye también en este ítem que las TIC son una herramienta que fomenta la ludificación y la estimulación a través del juego. Se muestran a continuación algunos ejemplos textuales sobre este tema: 
- "Las herramientas tecnológicas son una gran fórmula para motivar al alumnado y despertar su creatividad y, por consiguiente, su talento, ya que, son herramientas que ofrecen múltiples posibilidades para desarrollar la imaginación de los estudiantes" (Participante 9, curso 16-17).

- "Con las TIC, el alumno se está divirtiendo y jugando, al tiempo que aprende de modo efectivo, lo que es fundamental para su proceso creativo" (Participante 45, curso 17-18).

Figura 2. Potencialidades de las TIC mencionadas por los encuestados

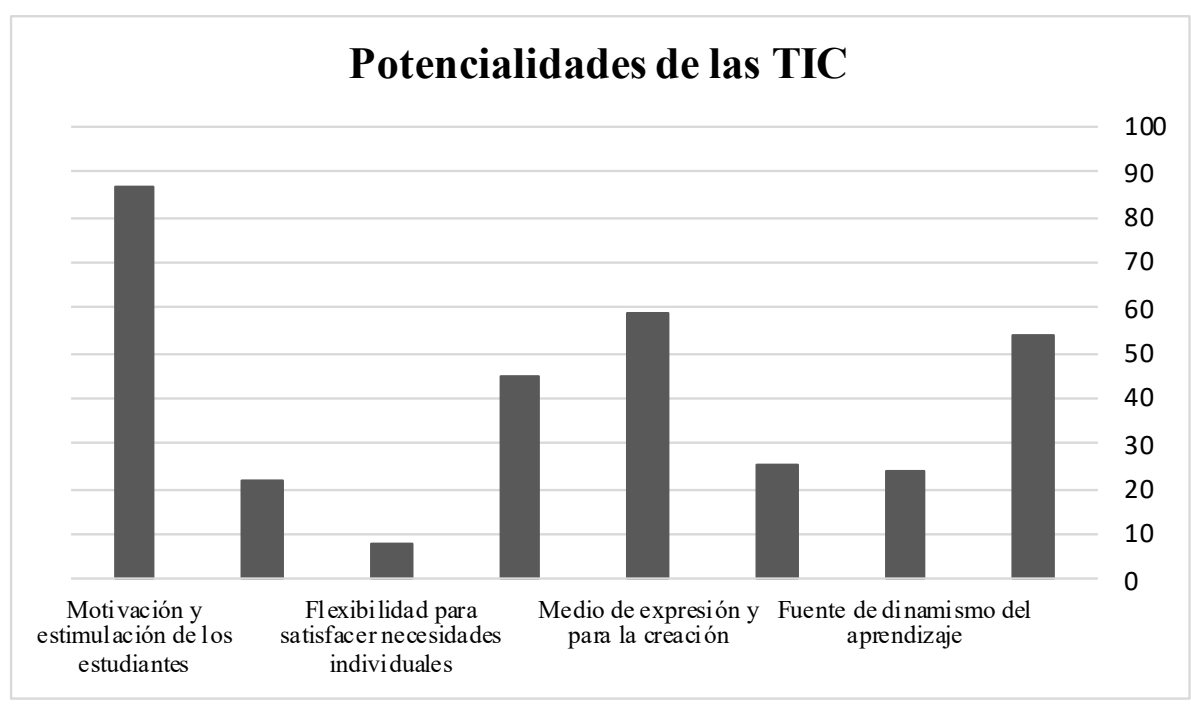

Fuente: Elaboración propia

También son destacables las reseñas "medio de expresión y para la creación" y "canal de comunicación e intercambio", siendo una ventaja tanto para el alumno como para la innovación del proceso docente:

- "Las TIC actualmente están inmersas dentro del modo de vida de la mayoría de nuestros alumnos (móviles, ordenador con internet, tabletas...) y qué mejor manera que enseñarles utilizando aquello que les motiva y saben manejar. En este sentido, las TIC pueden ser un medio para que los niños se puedan expresar y puedan ser capaces, cada vez con menos esfuerzo, de crear sus propios materiales e ideas" (Participante 21, curso 17-18).

- "Las nuevas tecnologías pueden usarse para ser un estímulo más que ayuden a desarrollar las competencias. Un ejemplo de ello sería hacer nuestro propio canal 
de YouTube con los alumnos y animarlos a que creen contenidos para subirlos luego a la web" (Participante 45, curso 15-16).

- "Las TIC pueden ayudar a fomentar la creatividad. Si analizamos los distintos ambientes de aprendizaje vemos que, en los nuevos modelos pedagógicos, el uso e intercambio de la información es vital. Y en esto las TIC juegan un papel relevante" (Participante 135, curso 17-18).

El ítem "fomento del aprendizaje colaborativo", donde los alumnos tienen que trabajar en equipo e interactuar para conseguir un objetivo común, fue el cuarto más citado:

- "Las nuevas tecnologías suponen una herramienta fundamental para potenciar la creatividad y el talento de las personas, ya que favorecen habilidades como la percepción, la retención de imágenes y palabras, la capacidad de atención y el trabajo cooperativo" (Participante 8, curso 17-18).

- "Las nuevas tecnologías multiplican las posibilidades del aprendizaje colaborativo y fomentan la comunicación, la interacción y el intercambio de ideas entre los miembros del grupo" (Participante 5, curso 15-16).

Son similares el número de referencias que se recogen sobre los aspectos de "generación de nuevos espacios interactivos y nuevas formas de presentar la información", "fuente de dinamismo del aprendizaje" y "acceso inmediato a información”. Como muestra se encuentran:

- "Las tecnologías tienen un gran poder en el proceso de enseñanza y aprendizaje. Los estudiantes pueden llegar a descubrir qué tan creativos pueden ser. En lugar de escribir un trabajo en papel, pueden escribir una entrada de blog, o en lugar de crear proyectos que se cuelgan de las paredes del colegio, subirlos a una página web" (Participante 9, curso 16-17).

- "Estamos hablando de una cantidad de estímulos y herramientas ilimitada. Un abanico de posibilidades que tiene el alumno del que antes no disponía" (Participante 52, curso 15-16).

- "Tenemos acceso a multitud de fuentes de información en diversos formatos: visual, escrito, sonoro... tal diversidad y riqueza nos permite sumergirnos en entornos a los que no tendríamos acceso sin la web. A través de internet, podemos recurrir a herramientas creativas adaptadas a nuestras capacidades, $o$ que a su vez nos permitan aprender y adquirir nuevas capacidades para crear" (Participante 26, curso 17-18).

Y, por último, los participantes destacaron de estas herramientas su "flexibilidad para satisfacer las necesidades individuales". Este ítem se refiere tanto a la 
posibilidad de adaptar horarios y lugares de conexión como a la ayuda para alumnos con necesidades educativas diferenciadas:

- "La flexibilidad de horarios para realización de tareas y la posibilidad de realizarlas desde su propio domicilio ayuda a fomentar el trabajo en grupo sin necesidad de desplazarse" (Participante 1, curso 15-16).

- "La tecnología puede ayudar a acortar la forma de educar, minimizar tiempos de estudios, investigación o simplemente desplazamientos innecesarios" (Participante 46, 2017).

- "La inclusión de las TIC en el trabajo diario de aula se considera como una medida ordinaria dentro del plan de atención en algunas comunidades autónomas, puesto que ofrecen apoyo visual y múltiples herramientas que facilitan el trabajo de docentes y alumnos" (Participante 58, curso 17-18).

\section{Competencia digital docente y otras categorías del uso de las TIC}

Finalmente, además de las potencialidades de las TIC ya analizadas, se han examinado otros aspectos comentados con asiduidad por los profesores que indicaron que las TIC fomentan la creatividad de los estudiantes. Así, se recogen en la tabla 1 las categorías en las que se agruparon estas referencias.

A continuación, igual que se ha hecho con el análisis de las potencialidades de las TIC, se recogen los nuevos ítems descritos y una serie de reseñas textuales para su ejemplificación.

La mayoría de los profesores comentaron cómo "la competencia digital docente" resulta fundamental para conseguir los objetivos propuestos y obtener unos resultados acordes con lo esperado si se llevan a cabo actividades con recursos digitales:

- "Es necesario decidir qué tipo de herramienta tecnológica contribuirá a desarrollar la capacidad creativa de nuestros alumnos, puesto que existen múltiples cuya finalidad puede variar considerablemente en función del uso que se haga de ellas" (Participante 3, curso 16-17).

- "Es necesario que, profesores y familias, estemos familiarizados y al tanto de este mundo virtual que nos rodea, ya que por mucho que le demos una tableta a nuestros alumnos, es cierto que lo verán atractivo, pero si no la utilizamos correctamente, no desarrollaremos su auténtico potencial" (Participante 2, curso 17-18).

En este proceso indicaron también que "las TIC son un apoyo", no son la única herramienta para conseguir potenciar la creatividad de los estudiantes: 
- "Las tecnologías nos proporcionan herramientas con las cuales, como docentes, podemos intentar potenciar la creatividad y el talento de los alumnos, pero no son indispensables para ello, aunque está claro que podemos sacar gran beneficio de ellas. Como educadores, debemos usarlas siempre como apoyo y no como algo insustituible y obligatorio" (Participante 42, curso 15-16).

- "Deben de ser un apoyo al aula y al aprendizaje y no pueden convertirse en el eje vertebrador ni en el andamiaje de la enseñanza. ¿Qué pasaría si, por ejemplo, un centro no dispone de los medios necesarios para utilizar las TIC asiduamente en el aula? ¿Los alumnos no se pueden desarrollar?” (Participante 11, curso 16-17).

Tabla 1. Otras categorías del uso de las TIC destacadas en el estudio

\begin{tabular}{|l|r|}
\hline Categorías & Referencias textuales \\
\hline Competencia digital docente (CDD) & 113 \\
Las TIC como apoyo & 92 \\
Necesidad de un cambio educativo & 88 \\
Uso de las TIC en la vida no académica & 52 \\
\hline
\end{tabular}

Fuente: elaboración propia.

Los docentes mencionaron que, para la inclusión de las TIC en los centros educativos, prima la "necesidad de un cambio educativo" requerido por la sociedad y la necesidad de una renovación metodológica en la docencia:

- "Los tiempos cambian y las metodologías que eran eficaces en antaño ya no lo son. Debemos movernos a la par cogiendo en todo momento lo que mejor se adapte al nivel y la edad de los alumnos, como las TIC" (Participante 41, curso 16-17).

- "La escuela tal y como la conocemos hoy en día ha quedado obsoleta y está pidiendo un cambio a gritos. Las TIC pueden ser el cambio que estaba demandando la sociedad educativa a nivel general, para ajustarnos a la nueva sociedad en la que vivimos" (Participante 14, curso 18-19).

La necesidad de cambio está, además, relacionada con el "uso de las TIC en la vida no académica", ya que estas tecnologías se encuentran en nuestro entorno cotidiano y son necesarias en la vida profesional:

- "Las TIC serán la base de cualquier tipo de enseñanza, ya que al igual que en el resto del mundo en los próximos años, las nuevas tecnologías serán la base en torno a la que girarán muchos aspectos de nuestra sociedad" (Participante 18, curso 17-18). 
- "Las tecnologías de la educación y la comunicación son imprescindibles en el entorno educativo porque están cada vez más presentes en nuestro entorno social, cultural, económico y laboral” (Participante 73, curso 17-18).

\section{DISCUSIÓN Y CONCLUSIONES}

Respecto al análisis de las características de los participantes en el estudio, con el aumento de edad hay una autopercepción en la disminución de la "capacidad de generar nuevas ideas" e "iniciativa y espíritu emprendedor", que puede haber sido condicionado por la metodología aplicada en el transcurso de su aprendizaje, e incluso posteriormente, adquirida en su proceso de enseñanza. Siendo el ítem de "capacidad para diseñar soluciones alternativas a problemas", directamente relacionado con el proceso creativo, el peor valorado por los participantes en todos los rangos de edades. Por tanto, como pudo comprobarse con el autoanálisis de las competencias del profesorado, existe una brecha digital intergeneracional en el manejo de las TIC entre los docentes encuestados a medida que aumentaba el rango de edad analizado. También se mostró una diferenciación por edades en los ítems que están relacionados con el propio concepto de la creatividad.

Con respecto a la pregunta central del estudio cayudan las herramientas tecnológicas a potenciar la creatividad de los estudiantes? como era de esperar, la mayoría de los docentes participantes en el análisis (85\%) resaltaron con sus intervenciones que las TIC promueven el desarrollo de la creatividad en los alumnos. Los datos son acordes con la bibliografía consultada que confirma que las nuevas tecnologías tienen mucho que ofrecer al mundo del intercambio educativo (Angarita, 2019; Das, 2019; Henriksen, Mishra y Fisser, 2016; Loveless, 2002), dado que permiten aplicar nuevos modos de presentar contenidos, búsqueda de información e investigación. Son precisamente las nuevas formas de aprender y el mayor acceso al conocimiento lo que ayuda a los discentes a ser más creativos. Un $2 \%$ de los participantes lo negaron rotundamente, argumentando la existencia de sobreestimulación, ya que tanto exceso de información y medios anulan en cierta medida la creatividad del niño, haciendo que todo se vuelva rutinario y aburrido. Llegaron incluso a considerar las TIC como una fuente de aislamiento social. Y un $13 \%$ de los docentes tuvieron una posición neutral, alegando que las herramientas tecnológicas y la creatividad son independientes, el uso de las tecnologías no está ligado a la creatividad ni al éxito. Las tecnologías son una herramienta más, pues se puede ser creativo manteniéndose desligado de ellas.

En relación con la pregunta ¿qué potencialidades de las TIC son las que más valoran dentro del ámbito educativo y cómo las relacionan con la creatividad?:

- "La motivación y la estimulación de los estudiantes" son los aspectos que más reiteran para que el aprendizaje sea más atractivo y capte la atención del alumno. Ítem que está íntimamente relacionado con que las TIC son un "medio 
de expresión y para la creación”. Esto está acorde con trabajos previos, como el de Jorda Lueges y Martínez Vázquez (2015), que en su estudio mostraron cómo ante condiciones favorables y estimulantes se permite la creación de productos, servicios, ideas y estrategias novedosas en una diversidad de campos; y el de Phutela y Dwivedi (2019), que puntualizan que las TIC en educación se utilizan para mejorar la creatividad, la interacción y el intercambio de conocimientos, aparte del aprendizaje de los estudiantes.

- Y también, según las aportaciones recogidas, las TIC constituyen un "canal de comunicación e intercambio", coincidiendo con diversos autores, como Carrió Pastor (2007) que señala que las TIC fomentan la relación entre alumnos y profesores de otros centros, favoreciendo una comunicación activa, abierta y natural tanto de forma síncrona como asíncrona, a través de correo electrónico, chats, foros, etc. que permiten compartir ideas, resolver dudas o enviar trabajos y actividades desde cualquier lugar y en cualquier momento. De la misma manera, estas tecnologías mejoran la comunicación entre alumnos y su aprendizaje cooperativo a través de actividades grupales (Gutiérrez-Porlán, Román-García y Sánchez-Vera, 2018). El uso de herramientas que faciliten la comunicación, la colaboración y la producción del conocimiento son indispensables para mejorar los procesos formativos (Suárez Guerrero y Gros Salvat, 2013).

- Otro elemento destacado fue el "fomento del aprendizaje colaborativo". Recordar en relación con el trabajo colaborativo que, dentro de las características que los participantes se autoevaluaron, el ítem de "capacidad para resolver problemas de forma colaborativa" de su valoración, fue de los mejor puntuados. Señalan además que la opción de una enseñanza colaborativa mediante el uso de herramientas tecnológicas ofrece muchas ventajas: favorece los diferentes ritmos de aprendizaje, atendiendo a las características y necesidades de nuestro alumnado, convierte a los estudiantes en protagonistas de su propio aprendizaje, desarrolla sus competencias y habilidades, refuerza sus relaciones interpersonales y les permite adquirir un aprendizaje significativo. En concordancia con el trabajo de Laisema y Wannapiroon (2014) que indicaban que las TIC fomentan las habilidades de pensamiento creativo porque los estudiantes pueden compartir conocimientos e interactuar con sus amigos. La creatividad se potencia ya que impacta positivamente en la producción y el procesamiento de múltiples alternativas (Lappas y Fessakis, 2014).

En resumen, las TIC se señalan como uno de los elementos clave del cambio social. Esta influencia se manifiesta, como indican, en los ámbitos económicos, sociales, políticos y educativos, y en que las nuevas tecnologías conviven en simbiosis con la sociedad actual. No cabe duda, por los resultados extraídos, de que las TIC se transforman en "compañeros de aprendizaje" de los estudiantes y en elementos imprescindibles de su vida laboral. Estas herramientas se convierten en el nuevo mediador de todos los procesos implicados en el aprendizaje, permitiendo la 
evolución de estrategias de autorregulación del alumno. Es por ello, por lo que se debe construir un ambiente de aprendizaje que estimule la creatividad de manera más efectiva a través de las interacciones entre los alumnos y estas herramientas de aprendizaje.

En este trabajo, por tanto, se destacan dos conclusiones obtenidas de las percepciones del profesorado, por un lado, que la creatividad es importante, y por el otro, que las TIC tienen el potencial de impactar y cambiar los procesos creativos.

En todos los casos, se destaca que es al docente a quien le corresponde crear un ambiente favorable e innovador que fomente el trabajo en equipo, brindando un clima agradable que propicie el proceso de aprendizaje. Los datos analizados ponen de manifiesto que los profesores son conscientes de que para propiciar una educación acorde a nuestro tiempo se deben realizar nuevas propuestas didácticas que introduzcan las herramientas necesarias para este fin, reforzando para ello nuevas habilidades y competencias en el desarrollo de la creatividad. Como señalan Csikszentmihalyi y Wolfe (2014) la creatividad en las escuelas es el resultado conjunto de la accesibilidad a la información, existencia de estudiantes interesados y maestros receptivos ante las ideas novedosas.

En este punto señalan que, además de formar a los alumnos en un buen uso de las nuevas tecnologías, ellos mismos deberían al mismo tiempo, recibir una buena formación para enseñar de forma óptima y ventajosa. Recogiendo en este sentido, todos los aspectos que engloban el concepto de la competencia digital docente (CDD). Se busca para ello el desarrollo de la CDD, que va mucho más allá de saber cómo usar las tecnologías, pues supone poseer conocimientos y capacidades para poder llevar a cabo procesos de selección e integración curricular de estas tecnologías (Prendes Espinosa, Gutiérrez Porlán y Martínez Sánchez, 2018). Las competencias digitales del profesorado son muy relevantes en el desarrollo de procedimientos de aprendizaje que introduzcan las tecnologías como herramientas al servicio de la educación (Fernández-Cruz y Fernández-Díaz, 2016). La CDD se ha convertido, por tanto, en un aspecto esencial en la formación de los profesores entendiéndola a modo de resumen, como el conjunto de conocimientos, capacidades y actitudes que los docentes deberían adquirir para responsabilizarse en garantizar una educación de calidad, que potencie el desarrollo del talento.

Las limitaciones de este estudio a pequeña escala incluyen (1) cómo entienden los docentes el concepto de "creatividad", y (2), cómo se identifica el papel de las TIC a través del sistema de categorías creado.

Los resultados de este artículo pueden tener implicaciones para que las instituciones sean conscientes de las opiniones de los docentes. Para estrechar la brecha digital entre el alumnado y profesores y progenitores es necesario mejorar la formación en el uso de las TIC y lograr un cambio en el enfoque didáctico que transforme la práctica docente, de modo que no se exploren las TIC como herramientas de trabajo que mejoren el método didáctico tradicional, sino que se integren de modo holístico en los planes de estudio. 


\section{REFERENCIAS}

Angarita L. (2019). Are ICT good partners for the development of creativity? A systematic review of literature. International Journal of Arts and Technology, 11(3), 112-123.

Betancourt Morejón, J., y Valadez Sierra, M. D. (2009). ¿Cómo propiciar atmósferas creativas en el salón de clases? Revista Digital Universitaria, 10(12). Recuperado de http://www.revista.unam.mx/vol.10/ num12/art85/int85.htm

Carrió Pastor, M. L. (2007). Ventajas del uso de la tecnología en el aprendizaje colaborativo. Revista Iberoamericana de Educación, 41, 1-10.

Coll, C. (2011). Aprender y enseñar con las TIC: expectativas, realidad y potencialidades. En R. Carneiro, J. C. Toscano y T. Díaz, T. (Coord.), Los desafíos de las TIC para el cambio educativo. Colección Metas Educativas 2021. OEI y Fundación Santillana.

Creely, E., Henderson, M., y Henriksen, D. (2019). Failing to succeed: The value of failure in creativity. En K. Graziano (Ed.), Proceedings of Society for Information Technology \& Teacher Education International Conference (pp. 1403-1411). Las Vegas, NV, United States: Association for the Advancement of Computing in Education (AACE).

Csikszentmihalyi, M. y Wolfe, R. (2014). New Conceptions and Research Approaches to Creativity: Implications of a Systems Perspective for Creativity in Education. In The Systems Model of Creativity, 161-184. Springer Netherlands.

Das, K. (2019). The Role and Impact of ICT in Improving the Quality of Education: An Overview. International Journal of Innovative Studies in Sociology and Humanities, 4(6), 97-103.

Ehtiyar, R., y Baser, G. (2019). University education and creativity: An assessment from students' perspective. Eurasian
Journal of Educational Research, 8o, 113-132. $\quad$ https://doi.org.10.14689/ ejer.2019.80.6

Fernández-Cruz, F. J., y Fernández-Díaz, M. J. (2016). Generation Z's Teachers and their Digital Skills. Comunicar, 46, 97-105. http://dx.doi.org/10.3916/C462016-10

Ferro Soto, C., Martínez Senra, A., y Otero Neira, M. C. (2009). Ventajas del uso de las TIC en el proceso de enseñanza-aprendizaje desde la óptica de los docentes universitarios españoles. Revista Electrónica de Tecnología Educativa, 29. https://doi.org/10.21556/ edutec.2009.29.451

Giménez, A. M., Luengo, J. A., y Bartrina, M. J. (2017). ¿Qué hacen los menores en Internet? Usos de las TIC, estrategias de supervisión parental y exposición a riesgos. Electronic Journal of Research in Educational Psychology, 15(3), 533-552. http://dx.doi.org/10.14204/ ejrep.43.16123

Gutiérrez-Porlán, I., Román-García, M., y Sánchez-Vera, M. (2018). Estrategias para la comunicación y el trabajo colaborativo en red de los estudiantes universitarios. Comunicar, 54(26), 91-100. https://doi. org/10.3916/C54-2018-09

Henriksen, D., Henderson, M., Creely, E, Ceretkova, S., Černochová, M., Sendova, E., Sointu, E. T., y Tienken, C. T. (2018). Creativity and Technology in Education: An International Perspective. Technology, Knowledge and Learning, 23, 409-424. https://doi.org/10.1007/s10758-0189380-1

Henriksen, D., Mishra, P., y Fisser, P. (2016). Infusing Creativity and Technology in 21st Century Education: A Systemic View for Change. Journal of Educational Technology \& Society, 19(3), 27-37.

$\mathrm{Hu}$, W. y Adey, P. (2002). A scientific creativity test for secondary school 
students. International Journal of Science Education, 24(4), 389-403. https://doi. org/10.1080/09500690110098912

Jorda Lueges, G., y Martínez Vázquez, N. E. (2015). Uso de técnicas de creatividad en un Entorno Virtual de Enseñanza Aprendizaje. Campus Virtuales, 4(1) 6672.

Laisema, S., y Wannapiroon, P. (2014). Design of Collaborative Learning with Creative Problem-Solving Process Learning Activities in a Ubiquitous Learning Environment to Develop Creative Thinking Skills. 5th World Conference on Educational Sciences - WCES 2013. Social and Behavioral Sciences, 116, (pp. 3921-3926).

Lappas, D., y Fessakis, G. (2014). Fostering creativity in computer supported collaborative learning activities. Proceedings of 8th International Technology, Education and Development Conference (INTED2014), Valencia.

Loveless, A. (2002). Literature review in creativity, new technologies and learning. Futurelab Research report 4. Recuperado de https://telearn.archivesouvertes.fr/hal-00190439/document

Maldonado, G., García, J., y SampedroRequena, B. (2019). El efecto de las TIC y redes sociales en estudiantes universitarios. RIED. Revista Iberoamericana de Educación a Distancia, 22(2), 153-176. doi: http:// dx.doi.org/10.5944/ried.22.2.23178

Moguel Pérez, G. A., Michel López, P., y Torres Hernández, M. J. (2016). Uso de la tecnología para fomentar la creatividad en el aprendizaje de la geometría. Revista de Investigación Educativa de la Escuela de Graduados en Educación, 12.

Nikolopoulou, K. (2018). Creativity and ICT: Theoretical approaches and perspectives in school education. En T. A. Mikropoulos (Ed.), Research on e-Learning and ICT in Education, (87-100), New York: Springer.
Prendes Espinosa, M. P., Gutiérrez Porlán, I., y Martínez Sánchez, F. (2017). Competencia digital: una necesidad del profesorado universitario en el siglo XXI. RED Revista de Educación a Distancia, 56.

Phutela, N., y Dwivedi, S. (2019). Impact of ICT in Education: Students' Perspective. Proceedings of International Conference on Digital Pedagogies (ICDP) 2019. doi: http://dx.doi.org/10.2139/ssrn.3377617

Ramírez-Montoya, M. S., y García-Peñalvo, F. J. (2018). Co-creación e innovación abierta: Revisión sistemática de literatura. Comunicar, 54, 08-18. https://doi. org/10.3916/C54-2018-01

Rashid, R. A., y Rahman, M. F. A. (2014). Social networking sites for online mentoring and creativity enhancement. International Journal of Technology Enhanced Learning, 6(1), 34-45. https:// doi.org/10.1504/IJTEL.2014.060024

Sarmiento, J. A. (2017). Desarrollo del pensamiento crítico y creativo mediante estrategias interconectadas: estrategias de aprendizaje, lectura crítica y ABP. Gestión, competitividad e innovación, 5(2), 145-162.

Smilansky, J., y Halberstadt, N. (1986). Inventors versus problem solvers: An empirical investigation. Journal of Creative Behavior, 2O(3), 183-201. http:// dx.doi.org/10.1002/j.2162-6057.1986. tbo0436.x

Sokól, A., Figurska, I., y Blasková, M. (2015). Using the Internet to Enhance Teaching Process at Universities for the Development of Creativity Competencies. Procedia-Social and Behavioral Sciences, 186, 1282-1288. https://doi. org/10.1016/j.sbspro.2015.04.036

Stolaki, A., y Economides, A. A. (2018). The Creativity Challenge Game: An educational intervention for creativity enhancement with the integration of Information and Communication Technologies (ICTs). Computers \& 
Education, 123, 195-211. https://doi. org/10.1016/j.compedu.2018.05.009

Suárez Guerrero, C., y Gros Salvat, B. (2013). Aprender en red: de la interacción a la colaboración. Barcelona: UOC.

Tabuenca, B., Sánchez, J. J., y Cuetos, M. J. (2019). El smartphone desdela perspectiva docente: ¿una herramienta de tutorización o un catalizador de ciberacoso? Revista de Educación a Distancia, 1(59). https://doi. org/10.6018/red/59/01

Talebian, S., Mohammadi, H. M., y Rezvanfar, A. (2014). Information and Communication Technology (ICT) in Higher Education: Advantages, Disadvantages, Conveniences and Limitations of Applying E-learning to Agricultural Students in Iran. Procedia-
Social and Behavioral Sciences, 152, 300-305. $\quad$ https://doi.org/10.1016/j. sbspro.2014.09.199

Wei, H. C., Peng, H. y Chou, C. (2015). Can more interactivity improve learning achievement in an online course? Effects of college students' perception and actual use of a course-management system on their learning achievement. Computers \& Education, 83, 10-21. https://doi. org/10.1016/j.compedu.2014.12.013

Yeh, Y., Yeh, Y., y Chen, Y. H. (2012). From knowledge sharing to knowledge creation: A blended knowledge-management model for improving university students' creativity. Thinking Skills and Creativity, 7(3), 245-257. https://doi.org/10.1016/j. tsc.2012.05.004

\section{PERFIL ACADÉMICO Y PROFESIONAL DE LOS AUTORES}

María José Cuetos Revuelta. Profesora Titular. Doctora Cum Laude por la Universidad de León. Premio Extraordinario de doctorado. Tiene un Máster Universitario en Energías Renovables, un Máster Superior en Prevención de Riesgos Laborales y un Experto en Sistemas Integrados de Gestión. Sus áreas de investigación incluyen las preferencias de aprendizaje aplicadas a las ciencias experimentales y la tecnología educativa y prevención de riesgos laborales para la transformación social. ID: http://orcid.org/0000-0002-9555-8765

E-mail: mjose.cuetos@unir.net

Dirección:

Universidad Internacional de La Rioja Avenida de la Paz, 137, Logroño, La Rioja (España)

Lucía Grijalbo Fernández. Está acreditada como Profesora Contratada Doctora. Doctora Cum Laude por la Universidad San Pablo CEU con un Máster Universitario en Gestión Integrada de la calidad, medio ambiente y prevención de riesgos laborales y un máster en Tecnología y Gestión de empresas del agua. Sus áreas de investigación incluyen la biorremediación de aguas y la tecnología educativa y la prevención de riesgos laborales para la transformación social. ID: http://orcid. org/0000-0002-3867-4911

E-mail: lucia.grijalbo@bureauveritas.com 
Elena Argüeso Vaca. Licenciada en Ciencias Ambientales por la Universidad de León. Es doctoranda en la Facultad de Educación de la Universidad de Alcalá de Henares de Madrid. Tiene el Máster Oficial en Formación del Profesorado, Educación Secundaria Obligatoria y Bachillerato, Formación Profesional y Enseñanza de Idiomas. Participa en la línea de investigación de la tecnología educativa y la prevención de riesgos laborales para la transformación social de Bureau Veritas Formación. ID: http://orcid.org/o0oo-0002-4569-3117

E-mail: elena.argueso@bureauveritas.com

Vanessa Escamilla Gómez. Coordinadora del Área de Medio Ambiente, Energía y Nuevas Tecnologías de Bureau Veritas Formación. Doctora en Químicas. Está acreditada como Profesora Contratada Doctora. Es Doctora Cum Laude por la Universidad Complutense de Madrid. Actualmente su línea de investigación se centra en la tecnología educativa y la prevención de riesgos laborales para la transformación social. ID: http://orcid.org/0000-0003-3790-2043

E-mail: vanessa.escamilla@bureauveritas.com

Ruth Ballesteros Gómez. Responsable Académica de Bureau Veritas Formación. Es Doctora europea Cum Laude por la Universidad Rey Juan Carlos. Tiene un Máster en Dirección Internacional de empresas, PRL y Petroquímica. Acreditada como Profesora Contratada Doctora. Colabora en la línea de investigación de la tecnología educativa y la prevención de riesgos laborales para la transformación social. ID: http://orcid.org/0000-0002-6327-7379

E-mail: ruth.ballesteros@bureauveritas.com

Dirección

Bureau Veritas Formación

Valportillo $1^{\text {a }}$, 22-24

Alcobendas, Madrid (España)

Fecha de recepción del artículo: 18/12/2019

Fecha de aceptación del artículo: 25/01/2020

Fecha de aprobación para maquetación: 07/03/2020 